Mots. Les langages du politique

Discours de violence au nom de la foi

\title{
Les fanatiques et le discours colonial
}

Paul Siblot

\section{OpenEdition}

\section{Journals}

Édition électronique

URL : https://journals.openedition.org/mots/1388

DOI : $10.4000 /$ mots. 1388

ISSN : 1960-6001

\section{Éditeur}

ENS Éditions

\section{Édition imprimée}

Date de publication : 1 novembre 2005

Pagination : 73-81

ISBN : 2-84788-084-4

ISSN : 0243-6450

\section{Référence électronique}

Paul Siblot, «Les fanatiques et le discours colonial », Mots. Les langages du politique [En ligne], 79 | 2005, mis en ligne le 28 mai 2008, consulté le 22 avril 2022. URL : http://journals.openedition.org/ mots/1388; DOl : https://doi.org/10.4000/mots.1388

\section{(c) ENS Éditions}




\section{Paul Siblot}

\section{Les fanatiques et le discours colonial}

Qui désigne-t-on par le mot fanatique? À cette question, les dictionnaires donnent des réponses convergentes dont Le Petit Robert (1993) propose la synthèse suivante : le fanatique 1. "se croit inspiré de la divinité, de l'esprit divin »; 2. est « animé envers une religion, une doctrine, une personne, d'une foi intraitable et d'un zèle aveugle»; 3. par extension, éprouve "une admiration, une passion excessive pour quelqu'un, quelque chose ». Le trait définitoire principal, pour l'ensemble des articles lexicographiques, est celui de croyances religieuses ou de convictions individuelles dont l'élévation au rang d'absolu conduit à l'outrance et à la violence.

\section{Aux origines de fanatisme}

mmLe terme a d'emblée été ancré dans le domaine confessionnel. Peut être qualifié de fanaticus ( $($, um) - selon l'étymologie, ce qui a partie liée à l'« espace consacré » pour un culte, fanum (de la racine italique *fes, *fas, à valeur religieuse). Cela concerne les servants qui ont en charge l'entretien des lieux et qui les protègent des profanations (littéralement, de l'intrusion des profanes auxquels l'espace hiératique est interdit). Certains de ces prêtres procèdent aux liturgies ; gardiens du temple au sens fort, ils tirent de leur étroite liaison avec le sacré une autorité ecclésiale qu'ils confortent par des manifestations destinées à marquer l'imagination des fidèles. Les prêtres de Bellone, d'Isis ou de Cybèle dansent et défilent en processions rituelles dans les rues, armés de glaives ou de haches, parfois nus, et dans le délire de la transe se mortifient, se blessent, s'estropient. On conçoit les réactions d'un public révulsé autant qu'impressionné, et l'on comprend que le rejet de ces outrances ait promu une étymologie sociale qui connote négativement les pratiquants des automutilations ${ }^{1}$. Une confirmation linguistique indirecte en est donnée dans le fait que l'adjectif ne désigne pas la fonction générale des ecclésiastiques, mais ce trait comportemental particulièrement saillant. Fanaticus est traduit, en même temps que défini, par « inspiré, rempli d'enthousiasme, exalté, en délire, frénétique » (F. Gaffiot). S’y

1. Voir M. Tournier, 2002 [1997, Paris, Klincksieck], "Fanatisme, de la fête à la fête », Propos d'étymologie sociale, t. 2, Lyon, ENSÉditions, p. 105-108.

Université Montpellier 3, Praxiling, ICAR, CNRS, paul.siblot@univ-montp3.fr 
ajoute par métaphore et substantivation la désignation d'un « arbre frappé de la foudre », laquelle illustre au plan concret le sens premier d'«illuminé » qui suppose une incidence du monde céleste. La gradation de l'exaltation qu'on suit dans la série des synonymes s'accompagne d'un désaveu implicite croissant, exprimé sans ambages dans la valeur adverbiale: fanatice, « en furieux ». L'empreinte des pratiques des fanatici et de leur désaveu dans le sémantisme du latin perdure en français.

L'entrée du mot dans le lexique se fait au $17^{\mathrm{e}}$ siècle (Académie, 1694) et la plus ancienne attestation répertoriée l'est en 1532 sous la plume de Rabelais :

Pantagruel reprend frère Jean, et luy dit: «Croyez que c'est la fureur poëtique du bon Bacchus [...] Et veu qu'il est de cerveau phanatique, ce me seroit acte trop piqueur, penser moquer un si noble trinqueur. » (Le Cinquième Livre, chap. 46).

L'ironie de l'occurrence littéraire, qui substitue l'inspiration des muses à celle de la transcendance, vise une exaltation due à de trop généreuses libations. L'usage courant maintient le terme dans le domaine religieux et la perte de raison imputée au fanatique y suit, comme en latin, une progression que balisent les synonymes répertoriés par le lexicographe: illuminé, intolérant, sectaire, exalté, extrémiste (toujours dans Le Petit Robert). La réprobation, absente de certaines valeurs prises par hyperbole dans d'autres domaines que religieux (fanatique de musique), croît avec l'intensification du comportement fanatique qui va jusqu'au délire. Au fil de la gradation, l'excès se voit de plus en plus nettement dénoncé: enthousiaste, fervent, passionné, mordu, enragé, fou. La reprise savante et tardive du terme en français fait qu'il n'apparait pas dans les chroniques ou les chansons de geste, ni dans la littérature épique où les Sarrasins ne sont pas fanatiques, bien que «suppôts de l'Antéchrist ». Non par défaut de qualificatif, qu'on aurait trouvé ou créé si on en avait éprouvé le besoin, mais en raison des valeurs d'une société pétrie de religiosité. La ferveur religieuse ne s'y infirme pas à devenir fureur, elle s'accroit au contraire des vertus du courage et de l'audace. Et cela vaut pour le protagoniste, ne serait-ce que par contrainte discursive : on ne peut dénigrer la figure de l'adversaire sans déconsidérer le mérite du combat et le prix de la victoire. En outre, dans l'esprit des Croisades comme dans celui du jihâd, la notion moderne de fanatique ne fait pas sens, a fortiori sa stigmatisation. L'évolution du sémantisme français résulte d'emplois postérieurs.

Les guerres de religion qui ravagent la France au $16^{\mathrm{e}}$ et au $17^{\mathrm{e}}$ siècles ne semblent pas, dans un premier temps, intéresser le mot dont l'usage ne se répand que lentement. En dépit du volume des écrits, de l'humanisme défendu et de l'horreur que les massacres de la Saint-Barthélémy (1572) inspirent à l'auteur, le propos de Montaigne ne comporte qu'une occurrence de fanatisme. C'est par la critique des pensées manichéennes qui séparent de façon catégorique le vrai et le faux, par le refus des jugements légitimant l'imposition de la «vraie foi » par tous les moyens, c'est à la suite de la défense de la tolérance et de l'engagement 
opiniâtre de Voltaire, c'est avec le discours des Lumières que l'usage de fanatisme entre dans les mœurs, avec une néologie de sens. L'article de Deleyre dans l'Encyclopédie (1751-1772) caractérise le fanatisme comme «superstition mise en action » et l'explique de la sorte : "Le fanatisme a pris naissance dans les bois, au milieu des ombres de la nuit et des terreurs paniques qui ont élevé les premiers temples du paganisme [...] [il] règne parmi ceux qui ont le cœur droit et l'esprit faux. » Imputé au retard des consciences dans l'intelligence du monde, le fanatisme devient un effet de l'intolérance et doit être combattu dans les esprits exaltés que des croyances viciées dévoient. La matrice de la signifiance de fanatique/fanatisme est alors posée et commande aux esprits éclairés de porter la lumière dans les univers mentaux obscurcis. L'acception vaut en français contemporain.

Par le biais des exemples, les dictionnaires signalent encore une chose. Quelques citations, reprises du Grand Robert (1977), le montrent immédiatement :

Les trembleurs [les quakers], gens fanatiques qui croient que toutes leurs rêveries leurs sont inspirées... » (Bossuet)

Les extases sans fin des Brahmes fanatiques. (Baudelaire)

Montrevel ne trouva pas les Fanatiques si aisés à réduire qu'il l'avait cru. On leur avait donné ce nom, parce que chaque troupe considérable de ces protestants révoltés avait avec eux quelque prétendu prophète ou prophétesse. (Saint-Simon)

Le domaine est bien celui du religieux mais les fanatiques, comme les barbares, ce sont toujours les autres. Il est rare qu'on applique aux siens et à soi-même cette hétéro-nomination; en raison de la composante dépréciative bien sûr, mais en raison logique également. Qu'on estime la conscience du fanatique obscurcie par la fureur qui l'emporte ou qu'on trouve aberrant son jugement jusqu'au-boutiste, jamais il ne peut se concevoir lui-même comme fanatique. N'est-ce pas une inaptitude à l'entendement qui précisément le caractérise? Et il est malaisé de se qualifier d'un qualificatif qui, par principe, disqualifie celui qui qualifie.

Le fanatisme concerne, à l'évidence, l'altérité, et il est dans l'ordre des choses qu'il concerne l'univers arabo-islamique, altérité prévalente de l'Europe. Des siècles de confrontation entre monothéismes exclusifs, de rivalités et d'affrontements en Terre sainte, dans la péninsule lbérique, sur la Méditerranée ou son pourtour, ne pouvaient pas ne pas promouvoir de représentations antagonistes. Au $18^{\mathrm{e}}$ siècle, il n'y a pourtant pas de relation préférentielle du fanatisme à l'aire arabo-musulmane. Et en 1793, les fanas, ce sont les monarchistes. L'occitan a depuis longtemps repris assacis, assassim de l'arabe, où il désigne des sectateurs ismaéliens (chiites) aux pratiques violentes; mais les troubadours en ont fait un qualificatif élogieux du vocabulaire amoureux (cf. œillade assassine). Le français a suivi - halsasin (1195) - mais le rapprochement d'assassin avec haschischin est plus tardif (Silvestre de Sacy, 1809) et reste discuté. Certes, Voltaire pourfend chez «le Grand Turc » un parangon du despotisme, 
et il commet une pièce de théâtre dédiée au pape, Mahomet ou le fanatisme; mais en précaution retorse, car ce sont la monarchie absolue et l'accointance entre pouvoirs spirituels et temporels qui sont visés, où qu'ils sévissent. À l'inverse, l'Empire ottoman lui sert de contre-exemple fréquent pour dénoncer les infirmités de la société européenne, et ce n'est pas à l'encontre du monde musulman qu'il porte au chapitre du fanatisme ses accusations les plus fortes. Ainsi dans l'article de son Dictionnaire philosophique (1764):

Le plus grand exemple de fanatisme est celui des bourgeois de Paris qui coururent assassiner, égorger, jeter par les fenêtres, mettre en pièces, la nuit de la Saint-Barthélémy, leurs concitoyens qui n'allaient point à la messe.

Sa récusation de l'intolérance et du fanatisme est universelle, et si la rhétorique tire parti des différences culturelles, elle n'est pas de parti pris ; les illustrations que Voltaire prend à l'altérité restent le plus souvent une prudence. II faut l'expédition d'Alger, la préparation de l'opinion par les publicistes de Charles X, les panégyriques sur la prise de la ville puis les commentaires qui célèbrent la conquête et la colonisation du pays, pour que s'installe au $19^{\mathrm{e}}$ siècle une association privilégiée qui fait de fanatisme musulman un syntagme en voie de figement. L'abondante phraséologie du Trésor de la langue française pour les collocations (dévotion, religion fanatique; nationalisme, patriotisme fanatique; fureur, haine, intolérance fanatique; amour, attachement fanatique. Fanatisme brutal, farouche, féroce; fanatisme catholique, chrétien, militaire, national, patriotique, républicain...) ne relève pourtant pas de liien entre arabe et musulman, en dépit de la profusion des attestations factuelles. La chose surprend et appelle une explication. Puisque les articles lexicographiques restent muets, il faut consulter le corpus historique ou, du moins, un échantillon des discours qu'il rassemble.

\section{Fanatisme musulman}

Les exemples retenus viennent des premiers acteurs, les militaires de la conquête de l'Algérie. Le premier est extrait de la correspondance du capitaine Lucien-François de Montagnac, dont 128 lettres furent publiées en 1885 . SaintCyrien intrépide, en campagne depuis 1836 , il croit pouvoir surprendre Abd elKader et part à sa rencontre à la tête de quatre cents soldats mais tombe sur plus forte partie. Au terme de trois jours de combats, la colonne est anéantie. Le nom et la date du fait d'armes - Sidi Brahim, 1845 - sont inscrits au mémorial de la conquête et, deux ans plus tard, on contraindra l'émir à faire acte de reddition sur les lieux du combat:

(Djemaa-Ghazaouet, le 7 juin 1845). Le caïd d'Oujda est remplacé par un autre. L'ancien caïd ne convenait pas à l'empereur'2 , qui le trouvait trop facile à notre endroit,

2. Il s'agit du sultan du Maroc. 
et il est probable qu'on a désigné pour le remplacer un vieux reste de fanatisme musulman 3 .

Plusieurs informations peuvent être retenues. D'abord celle d'un emploi banal, qui va de soi et n'appelle pas de glose. Cette occurrence unique signale ensuite que si l'usage en est ordinaire, le terme n'a pas pour autant de fréquence particulière. S'affiche enfin la condescendance, sinon le dédain pour un univers caractérisé par l'arriération et l'obscurantisme.

La seconde illustration est plus prolixe. Les textes, nombreux, rassemblent une correspondance abondante et plusieurs volumes de mémoires où le terme se révèle récurrent. Arrivé lieutenant en Algérie, Achille Le Roy de Saint-Arnaud en repart général, mais c'est à Paris qu'avec le coup d’État du 2 décembre 1851, il gagne son bâton de maréchal. Cet «Africain», que l'hagiographie cite en exemple ${ }^{4}$, relate au quotidien les razzias qu'il conduit à la tête de ses « colonnes de feu ». L'emploi de fanatique/fanatisme y est courant ${ }^{5}$ :

(Camp du Fondouk, le 20 juin 1838.) Les Bédouins continuent à couper les têtes. On attribue ce passe-temps à des coupables isolés, étrangers à la plaine, descendus des montagnes pour aider aux moissons et que leur fanatisme a poussés contre nous.

(Milianah, le 28 mars 1843.) Voilà la guerre d'Afrique ; on se fanatise à son tour et cela dégénère en une guerre d'extermination.

(Orléansville, le 6 août 1845.) Bou-Maza, selon les circonstances, dit aux Arabes qu'il travaille pour l'Émir ou pour lui. C'est un homme adroit, entreprenant, audacieux, et qui décidément exploite bien le fanatisme des Arabes.

(Au bivouac d'Aïn-Méran, le 15 août 1845.) J'ai rejeté Bou-Maza sur les colonnes de Ténès et de Mostaganem... Il a fini par s'échapper... On m’a rapporté trente-quatre têtes, mais c'est la sienne que je voulais. Le même jour je poussais une reconnaissance sur les grottes ou plutôt les cavernes: deux cents mètres de développement, cinq entrées. Nous sommes reçus à coups de fusil... Le 9 commencement des travaux de siège, blocus, mines, pétards, sommations, instances, prières de sortir et de se rendre. Réponses : injures, blasphèmes, coups de fusils... feu allumé. 10, 11, même répétition. Un Arabe sort le 11, engage ses compatriotes à sortir; ils refusent. Le 12 onze Arabes sortent, les autres tirent des coups de fusil. Alors je fais hermétiquement boucher toutes les issues et je fais un vaste cimetière. La terre couvrira à jamais les cadavres de ces fanatiques. Personne n'est descendu dans les cavernes; personne à part moi ne sait qu'il y a là cinq cents brigands qui n'égorgeront plus les Français.

(Orléansville, le 13 avril 1847.) Bou-Maza n'est pas un homme ordinaire. Il y a en lui une audace indomptable jointe à beaucoup d'intelligence, dans un cadre d'exaltation et de fanatisme ${ }^{6}$.

3. L.-F. de Montagnac, 1998 [rééd.], Lettres d'un soldat. Algérie, 1837-1845, Vernon, Christian Destremau.

4. Voir F. Maspero, 1993, L'honneur de Saint-Arnaud, Paris, Plon.

5. Dans les citations, l'italique est de l'éditeur.

6. Revue politique et littéraire, 1978 [1906], Paris, Laffont. 
Saint-Arnaud enregistre sans état d'âme ses campagnes et tient la comptabilité quotidienne de sa gloire. Il rapporte l'ordinaire de la conquête et en dresse la norme; dans ces récits guerriers, les emplois de fanatique sont associés au vocabulaire militaire et à un champ lexical macabre (couper les têtes, guerre d'extermination, trente-quatre têtes, coups de fusils, mines, sommations, vaste cimetière, cadavres, n'égorgeront plus...). La mort est son métier, c'est en professionnel que l'officier apprécie la technique de l'ennemi. Pour lui, le fanatisme ne saurait être condamné ; il est dans l'ordre des choses et participe des nécessités de la guerre. Admiratif de l'adversaire (adroit, entreprenant, audacieux, qui exploite bien, une audace indomptable, beaucoup d'intelligence), il se voit conduit à l'imiter et à " se fanatiser » lui-même; il concède qu'il y a bien quelque outrance à exterminer, mais ne désavoue pas la règle du jeu. La partie cependant n'est pas égale au plan du discours, car seul l'ennemi y est fanatique. La disparité présente un double avantage. S'il y a outrance et transgression dans la violence, celle que le narrateur se voit tenu de pratiquer n'est que juste retour des choses: la responsabilité en revient à l'autre, qui ne sait respecter de mesure. Par ailleurs, ce fanatique que la passion emporte hors de toute raison, et qui ne peut maitriser ses emportements, ne saurait être un interlocuteur. Le seul langage qu'il connaisse, le seul qu'on puisse lui faire entendre, est celui de la force brute qu'il exerce et qu'on se trouve dans l'obligation d'exercer à son encontre. Ainsi se voient légitimés le recours à la contrainte et les moyens de la conquête, y compris les «enfumades» de tribus entières, femmes et enfants compris ${ }^{7}$. La même logique explique que le chef des fanatiques n'en soit pas un, lui dont on reconnait l'intelligence militaire et le talent à manipuler les fanatiques.

Le fanatisme connait d'autres usages argumentatifs. On s'en tiendra à celui de Louis Veuillot, qui consigne son expérience algérienne dans une sorte de journal personnel mêlé de correspondances, paru en 1845, Les Français en Algérie. Souvenirs d'un voyage fait en 1841. Monarchiste convaincu et chrétien militant, il est proche du pouvoir ; il occupe les postes de secrétaire du gouverneur d'Algérie - alors le maréchal Bugeaud - puis de Monseigneur Dupuch, premier évêque d'Alger. À défaut d'être au combat, il vit sa mission en croisé :

On ne voit rien que de légitime à brûler les maisons des Arabes; on permet aux Maures de dire publiquement dans leurs mosquées la khotba au nom de l'empereur du Maroc et même au nom d'Abd el-Kader, mais on interdit aux prêtres catholiques toute démarche qui aurait pour but d'amener un musulman à se faire chrétien, et la raison, c'est qu'il ne faut pas exciter leur fanatisme ${ }^{8}$. (p. 58)

Les Turcs professaient la religion de leurs sujets, nous n'en professons aucune, et nous n'avons pas même la ressource d'opposer un fanatisme à un autre. (p. 119)

7. Ces procédés féroces suscitèrent quelques remous à la Chambre, qui arrêta la constitution d'une commission. Il n'y eut pas de suites.

8. L. Veuillot, Les Français en Algérie. Souvenirs d'un voyage fait en 1841, 1978 [186o], Paris, Laffont. 
Si nous parvenons à détruire Abd el-Kader, on pourra dire non seulement que nos armes ont fait un grand prodige, mais aussi que la foi musulmane s'est intensément affaiblie, et que ce peuple, fanatique encore, ne l'est plus assez pour fermer ses yeux et son oreille aux accents de la vérité. (p. 230)

Que fait-on pour s'assimiler ces populations fanatiques, assez promptes à oublier leur religion pour les avantages du trafic et du plaisir, mais plus promptes encore, lorsque les profits du commerce ont réparé leurs pertes, à courir aux armes, à la voix de qui représente à la fois chez eux l'indépendance et la religion? Que fait-on pour les attacher à la France, pour changer leurs idées et leurs mœurs, pour apaiser ce fanatisme redoutable? On ne fait rien; et, qui pis est, on ne veut rien faire. [...] Tant que les Arabes ne seront pas chrétiens, ils ne seront pas français, et tant qu'ils ne seront pas français, nul gouverneur, nulle armée ne pourra garantir pour un mois la durée de la paix. (p. 291)

Le propos est sans fard et ne manque pas d'une certaine lucidité. Il anticipe sur ce qui restera la question politique majeure de la colonie, restée sans réponse jusqu'au dénouement historique de l'accession de l'Algérie à l'indépendance, près d'un siècle et demi plus tard. C'est celui des modalités d' «insertion », d' « intégration », d' "assimilation »... des «indigènes », "sujets » mais non citoyens français. Le problème est celui du statut des colonisés, autrement dit du rapport colonial et de la colonie elle-même. Le sémantisme de fanatique demeure associé au vocabulaire de la guerre et de la violence (brûler les maisons, détruire, nos armes, nulle armée, la durée de la paix), mais secondairement. Le rapport principal est avec la religion (dire la khotba dans leurs mosquées, prêtres catholiques, amener un musulman à se faire chrétien, la religion [trois occurrences], foi musulmane, la vérité, chrétien) perçue comme le centre ultime de résistance qui doit être, en bonne stratégie, anéanti. Seule façon d’y parvenir, éradiquer la religion musulmane par évangélisation et conversion. Nullement condamné, le fanatisme de l'adversaire est au contraire envié et son absence dans le corps expéditionnaire déplorée. Signe de piété et de la toute puissance de la foi, il pourrait qualifier la radicalité du discours tenu si le terme n'était marqué de péjoration et associé à l'islam dans le discours ordinaire. Ce qui apparait à foison dans d'autres textes. À l'exemple de l'expédition d'Égypte, celle d'Alger entraine à sa suite une cohorte de savants chargés de dresser un état des lieux et de réunir les connaissances utiles sur l'Afrique. Le capitaine E. Carette coordonne une volumineuse Exploration scientifique de l'Algérie, 1844-1867; il y use volontiers de l'acception courante de fanatique:

C'est sous la domination des Zirites que les peuples chrétiens portèrent la guerre en Afrique. On sait que la puissance musulmane vint échouer au-delà des Pyrénées contre la bravoure française, qui imposa des limites à l'invasion de ces hordes fanatiques ${ }^{9}$. (p. 198)

9. E. Carette, s. d., Exploration scientifique de l'Algérie, 1844-1867, Bouslama [rééd.], Tunis) 
Du fond de sa retraite, dans le Maroc, Abd el-Kader envoyait des émissaires pour prêcher la révolte... Ces sourdes menées portèrent un coup funeste à la tranquillité du pays. Le 30 janvier [1845], une bande de fanatiques, excités par les prédications d'un marabout des Ouled-Brahim, se présenta sans apparence hostile devant le poste de Sidi Bel-Abbès dans la province d'Oran. Introduits dans le camp, ils tirèrent tout à coup des armes de dessous leur burnous et se précipitèrent sur nos soldats... En quelques instants les cinquante-huit fanatiques qui avaient pénétré dans le poste furent tous massacrés. (p. 330)

À quelque temps de là, nos troupes campées chez les Ouled Khiar, non loin de la frontière, furent attaquées, le 19 juin 1846, par un rassemblement de plusieurs milliers de cavaliers et de fantassins, conduits par un marabout. Notre cavalerie s'élança contre ces troupes de fanatiques, les dispersa et tua plus de cent hommes ${ }^{10}$. (p. 339)

On retrouve les associations avec le vocabulaire de la guerre (porter la guerre, la bravoure, l'invasion, la révolte, hostile, le camp, des armes, massacrés, attaquées, cavaliers et fantassins, tua plus de cent) et avec celui de la religion (chrétiens, puissance musulmane, prêcher, prédications d'un marabout, conduits par un marabout). Parallèles et récurrentes, elles participent de l'ordre de l'évidence et du sens commun. La répétition des syntagmes (hordes fanatiques, bande de fanatiques, troupes de fanatiques) signale un processus de stéréotypisation et la catégorisation, collective et indifférenciée, révèle une ethnicisation péjorante. Ce qu'on vérifie par défaut. Contrairement à l'usage, le capitaine Louis-Adrien Berbrugger évite sciemment cet emploi habituel de fanatique et ironise sur ceux qui en usent et en abusent. Ce militaire n'est pas à l'image de ses pairs; on le voit, lors de la prise de la smala, courir la campagne après les feuilles de la bibliothèque d'Abd el-Kader que les soudards ont jetées au vent. Et c'est lui qui réunit le premier fonds de ce qui deviendra plus tard la Bibliothèque nationale d'Algérie. Il poursuit ainsi le rêve saint-simonien d'«unir Orient et Occident», ce qui exclut d'invalider les partenaires par assignation de fanatisme. Il en est de même des romantiques en quête d'exotisme. Dans son Voyage pittoresque en Algérie (1845), Théophile Gautier n'use qu'à deux reprises du terme ; encore est-ce à propos des pratiques mortificatoires de la secte des Aïssaoua. Les quatre cents pages qu'Eugène Fromentin consacre à l'Algérie (Un été dans le Sahara, 1854; Une année dans le Sahel, 1858) ne comportent qu'une seule occurrence, pour citer le lieu commun d'un discours rapporté.

Dans ses usages comme dans ses refus, la stéréotypie du fanatique/fanatisme musulman révèle la diversité des positionnements des locuteurs au sein de la formation discursive coloniale. On peut discuter l'évaluation mais non la fréquence, indubitable et significative, des collocations. Faut-il attribuer l'omission des relevés lexicographiques à la péjoration ethnique dont le contexte

10. Extraits sous l'intitulé Algérie dans L'Univers ou Histoire et description de tous les peuples. Algérie, de leurs religions, mœurs, coutumes, etc. [s. d.], Paris, Firmin-Didot. 
colonial les a chargées ? Ce serait par censure. L'évaluation critique de la France coloniale au plan des institutions comme à celui de l'imaginaire collectif n'en étant qu'à ses prolégomènes, on ne peut a priori écarter l'hypothèse d'un embarras à reconnaitre l'inscription d'une vision raciale en langue française. Sans en exclure l'éventualité, une autre explication peut être avancée. Les discours de conquête correspondent à une période donnée. Une fois les sabres au fourreau et les militaires au cantonnement, il faut des modalités de cohabitation avec les "fanatiques ". Ce qui implique un modus vivendi, d'autres rapports et de nouvelles représentations, incompatibles avec l'entière disqualification des interlocuteurs. Par ailleurs, si le fanatisme musulman constitue un argument justificatif de la conquête, «l'œuvre civilisatrice » ne peut s'en accommoder durablement sous peine de devoir avouer son échec. La formation discursive coloniale est tenue d'opérer une recomposition qui condamne les catégorisations fanatique/fanatisme musulman et l'essentialisation qu'elles impliquent.

À l'interrogation initiale, on peut risquer des éléments de réponse qui ne seront pas d'ordre lexical (les définitions des dictionnaires sont adéquates), mais discursif. Le sens produit par l'actualisation des mots l'est en relation avec des éléments du contexte discursif, du contexte de communication et de l'interdiscours. Faute d'une prise en compte de ce dernier, les syntagmes fanatique/ fanatisme musulman ne peuvent être pleinement appréhendés dans l'artefact de leur isolement. Un extrait pris à l'article islam du Nouveau Larousse illustré (1906), représentatif de l'arrière plan discursif mobilisé, permet de repérer le fonctionnement :

La décadence intellectuelle de l'islam actuel étonne en face du développement menaçant de ses dogmes religieux. [...] Les sectateurs du Coran, qui ont transmis à l'Europe du Moyen Âge soit directement, soit par l'intermédiaire du rabbinisme, la culture philosophique de la Grèce, sont devenus les tributaires de l'Occident dans les plus minimes détails; mais malgré cela, malgré l'influence toujours croissante des sciences et des doctrines de l'Europe en Orient et en Afrique, le musulman garde intacte son hostilité traditionnelle contre le Franc; plus il s'assimile ses procédés et jusqu'à un certain point ses manières de penser et ses méthodes, plus il devient ennemi de cette civilisation, dont chaque détail ne peut manquer de blesser ses sentiments religieux. La seule chose qui permette aux nations européennes de ne pas s'inquiéter outre mesure de cette tendance de l'islamisme moderne est le fait que les nations musulmanes ne forment pas un tout soumis à l'autorité d'un même souverain.

Tel est le sens commun. Pas très exactement celui dans lequel est inscrit le fanatisme musulman de notre échantillon au regard de la date plus tardive de la citation (1906). Mais il suffit à donner une idée du dialogisme que la nomination fanatisme musulman entretient avec les discours de caractérisation du musulman. C'est dans ce cadre, celui de la formation discursive coloniale, qu'opère la production de sens. Son caractère daté et limité dans le temps est une explication de l'oubli lexicographique, non sa justification. 